\title{
Impact of knowledge management in supply chain management: a study in Malaysian manufacturing companies
}

\begin{abstract}
This research deals with two important aspects of Knowledge Management (KM) within the context of Supply chain Management (SCM): Knowledge Acquisition and Knowledge Application. Supply Chain Learning (SCL) and Applied Supply Chain Process Knowledge (Applied SCPK) are used as proxies for Knowledge Acquisition and Application. This study aims to test the relationship between Applied SCPK, SCL, and Organization Performance. This study also looks at the effect of Environment Knowledge as a moderating variable. About 1608 questionnaires were distributed to all the manufacturing firms in Malaysia. Path Analysis and ANOVA were used to study the various relationships. Results indicate existence of strong relationships between the variables. The combined effect of SCL and Applied SCPK on Organization Performance is more significant than looking at the effects separately. Furthermore, Environment Knowledge is found to have moderating effect on the relationship between Applied SCPK and Organization Performance. This paper demonstrates the application of KM in SCM and shows the effect on Organization Performance. This may guide supply chain managers to create an environment conducive to acquisition and application of knowledge.
\end{abstract}

